\title{
Characterization of extrusion flow using particle image velocimetry
}

\author{
J. E. Fournier, M. F. Lacrampe*, P. Krawczak \\ Ecole des Mines de Douai, Department of Polymers and Composites Technology \& Mechanical Engineering, 941 rue \\ Charles Bourseul, BP 10838, 59508, Douai, France
}

Received 29 May 2009; accepted in revised form 24 June 2009

\begin{abstract}
The aim of this study was the characterization of polymer flows within an extrusion die using particle image velocimetry (PIV) in very constraining conditions (high temperature, pressure and velocity). Measurements were realized on semi-industrial equipments in order to have test conditions close to the industrial ones. Simple flows as well as disrupted ones were studied in order to determine the capabilities and the limits of the method. The analysis of the velocity profiles pointed out significant wall slip, which was confirmed by rheological measurements based on Mooney's method. Numerical simulations were used to connect the two sets of measurements and to simulate complex velocity profiles for comparison to the experimental ones. A good agreement was found between simulations and experiments providing wall slip is taken into account in the simulation.
\end{abstract}

Keywords: rheology, wall-slip, particle image velocimetry, extrusion, polycarbonate

\section{Introduction}

Due to the severe pressure and temperature conditions involved during polymer processing, the tooling is made of steel or aluminum and gives little access to the inside of the mold cavity or the die. The consequence is an incomplete knowledge of the phenomena that can appear in this 'black box' especially concerning the way the flow develops and is affected by the processing conditions and the mold or die specificities. It is therefore interesting to develop and implement techniques that can give more information about what happens during the process and also to check the validity of the numerical models.

Several methods have been investigated to characterize the flow within an injection mold or an extrusion die. The basic one is based on direct observation of the flow through a transparent window that is generally made of glass or quartz in order to withstand pressure and heat. This approach was successfully used by Yokoi et al. [1-5] to study phenomena such as jetting, weld lines and flow marks formation. Dowling and Bress [6] and Nabialek [7] also used this technique to compare mold filling simulations to actual behavior. Unfortunately, this method provides mainly qualitative information about the flow front and gives no quantitative measurements that would describe the flow more precisely.

In order to get a better description of the flow, optical techniques have been adapted to polymer processing. They are commonly used in other engineering sectors such as aero- or hydrodynamics but are not extensively used in polymer processing due to the difficulty of implementation in conditions close to industrial ones. One of the main difficulties is that these methods generally request one or two optical accesses to the flow, which means molds or

*Corresponding author, e-mail: lacrampe@ensm-douai.fr (C) BME-PT 
dies with transparent walls withstanding very high pressure and temperature.

Most techniques are based on laser particle velocimetry with two main physical principles to determine the local velocity. The first one uses the Doppler effect to measure the speed of seeds that are inserted into the fluid (Laser Doppler Velocimetry - LDV). It was used by several authors [8-11] to study polymer flow features such as flow instabilities in extrusion molding, wall slip and die swell. This method is very precise and convenient for detailed analyses of some phenomena. It is however time consuming due to the measurement principle, one point after the other, and is therefore not suitable for the study of transient flows such as those occurring during injection molding.

The second group of methods is based on optical particle tracking with different variants such as particle tracking velocimetry (PTV), particle streak imaging (PSV) or particle image velocimetry (PIV). They normally need two optical accesses to the flow, one for laser lighting and the other for particle movement recording. All variants use seeds to make the flow visible, at rather low concentration for PTV and PSV in order to be able to follow the particles individually, at a higher concentration for PIV due to the global analysis of the pictures. Martyn et al. [12] used PSV and PTV to study the recombination of polyethylene flows within a die whereas Yokoi et al. [13] used the same kind of techniques to study the filling of a mold with polystyrene. However, if the PIV technique is being increasingly used in the analysis of flow kinematics of non-newtonian fluids, in particular in the study of flow instabilities and wall slip, it has been rarely used to study molten polymer in industrial configurations (materials, machines and molds) due to the unfavorable and very constraining environment imposed by polymer processing (high pressure and temperature). As a consequence, little work has been published concerning measurements on real engineering thermoplastics (such as polycarbonate for example). Nigen et al. [14] used this method to analyze flow instabilities of model polymers (liquid at room temperature) when passing a sudden die contraction.

The measurement method chosen for this study is PIV. The aim was to check its applicability and efficiency to characterize the flow in test conditions as close as possible to real processing ones i.e. using a semi-industrial extrusion die connected to an industrial extruder and fed with an engineering polymer (polycarbonate). High temperatures and pressures were therefore involved in the experiments. Simple flows in a rectangular die were investigated as well as disrupted ones generated by a flow restriction. The direct observations of actual flows were then compared to numerical simulations. Due to significant wall slip appearing in the PIV measurements, wall slip was also taken into account in the simulation through rheological measurements based on the classical Mooney's method.

\section{Experimental}

The material chosen for this project was polycarbonate (PC, Lexan ${ }^{\circledR}$ from GE Plastics). For a comparison purpose, an other polymer, namely a polystyrene (PS, HH999 from BP Chemicals) has been used punctually. The processing equipment consisted of a single screw extruder (Kaufman ${ }^{\circledR}$, screw diameter: $40 \mathrm{~mm}, L / D=22$ ) and a rectangular die specially developed for PIV measurements, connected to the extruder by an adaptation device. The die had a rectangular section $(6 \mathrm{~mm} \times 60 \mathrm{~mm})$ and a length equal to $200 \mathrm{~mm}$, and contained a quartz transparent window $(14 \mathrm{~mm} \times 80 \mathrm{~mm})$ on two orthogonal faces (Figure 1) in order to meet the requirements of the PIV method. In a second step, the basic geometry was slightly modified by fixing a small obstacle $(4 \mathrm{~mm} \times 8 \mathrm{~mm} \times 14 \mathrm{~mm})$ on the bottom surface of the die. The maximum flow rate of the extruder was $30 \mathrm{~kg} / \mathrm{h}$, which corresponds to a maximum average velocity of $20 \mathrm{~mm} / \mathrm{s}$ and a maximum apparent shear rate of $20 \mathrm{~s}^{-1}$. Melt and die temperatures were set at the same level (280 to $320^{\circ} \mathrm{C}$ for polycarbonate and $220^{\circ} \mathrm{C}$ for polystyrene). The temperature control system of the die was that of the industrial extrusion machine. It is therefore necessarily less efficient than the temper-

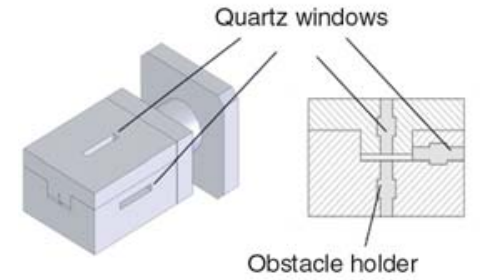

a) b)

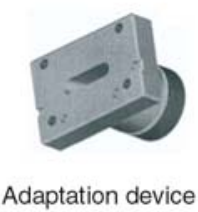

c)
Figure 1. Extrusion die: a) overview, b) transverse section, c) adaptation device 
ature control systems attached to laboratory devices such as capillary rheometers. That is why the experiments carried out on the industrial extruder have been always performed under the same conditions, after stabilization so as to reach a steady state and thus to limit this inconveniency.

PIV equipment was supplied by Dantec Dynamics. The principles of this measurement technique can be found elsewhere [15]. The lighting was generated by a Nd:YAG laser emitting at $532 \mathrm{~nm}$ (green). Optical lenses are used to create a $1 \mathrm{~mm}$ thick laser sheet. A CCD camera recorded the pictures. Aluminum powder (average diameter: $1.5 \mu \mathrm{m}$ ) was used as tracer at a weight concentra-

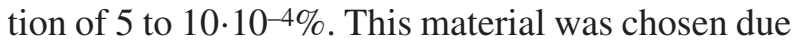
to its high light reflectivity that appeared adequate after comparison with others materials (talcum, glass spheres). The time between photographs was adapted to the extrusion flow rate and varied from 5000 to $30000 \mu \mathrm{s}$. The size of the interrogation window (IW) was kept constant, equal to $64 \times 64$ pixels (i.e. $558 \times 558 \mu \mathrm{m}$ ). The observation zone covered 25 IW resp. 11 IW in the flow direction resp. in the transverse direction. The choice of these parameters is the result of a compromise between the size of the gap to be observed, the image quality, the average displacement of the particles between two successive images and the mean flow rate.

Rheological measurements were carried out on a rheograph (Göttfert) using 0.5 and $1 \mathrm{~mm}$ capillary dies with a $L / D$ ratio of 20 . Wall slip was determined using the classical Mooney's method [17, 18]. For a capillary flow, it is based on Equation (1):

$\dot{\gamma}_{A}=\dot{\gamma}_{A, S}+\frac{4 V_{s}}{R}$

where $V_{s}$ is the slip velocity, $\dot{\gamma}_{A}$ the apparent shear rate (calculated from flow rate $Q$ and die radius $R$ by Equation (2)) and $\dot{\gamma}_{A, S}$ the apparent shear rate corrected for slip, which is only a function of the wall shear stress $\left(\tau_{w}\right)$.

$\dot{\gamma}_{A}=\frac{4 Q}{\pi R^{3}}$

At constant shear stress, the slip velocity can then be calculated from a plot of $\dot{\gamma}_{A}$ vs. $1 / R$, which is a straight line of $4 V_{s}$ slope. To apply this method, it is therefore necessary to measure the flow curves $\tau_{w}$ vs. $\dot{\gamma}_{A}$ for capillaries of various diameters.

Numerical simulations were performed using REM 3D software package supplied by Transvalor. This finite elements software is dedicated to the three dimensional simulation of extrusion and injection molding processes and their variants (co-injection, co-extrusion, gas and water assisted injection molding). It contains a specific module based on a power law model (Equation (3)) to introduce wall slip in the computation.

$\tau=\alpha \cdot K \cdot V_{s}^{n}$

with $\tau$ the shear stress, $\alpha$ the slip coefficient, $K$ the consistency, $V_{s}$ the slip velocity and $\mathrm{n}$ the exponent of the power law.

\section{Results and discussion}

Two slightly different geometries were analyzed. The first corresponded to a basic flow in a rectangular die. It was used to implement and validate the method, and set the measurement parameters. It was also used to study the velocity profile through the thickness of the die. In the second case, the flow was disrupted by means of a geometrical discontinuity. This obstacle generated an abrupt step in the flow and a complex deviation of the latter. The resulting velocity profile was then compared to numerical simulations introducing wall slip in the computation on the basis of rheological measurements.

\subsection{PIV measurements}

\subsubsection{Simple flows}

A typical example of a velocity field measured in the rectangular die for polycarbonate is presented in Figure 2. Figure 3 shows the profiles through the thickness of the die for the different flow rates that can be reached with the processing equipment. Though the velocity profiles appear globally as quadratic power laws as one could expect for Newtonian flows (which is the case of polycarbonate in the range of temperature and flow rates covered by this study) between parallel plates, several peculiarities can be seen on these profiles. First, some velocity profiles are not symmetric with respect to the axis of the die. However it is worth reminding 


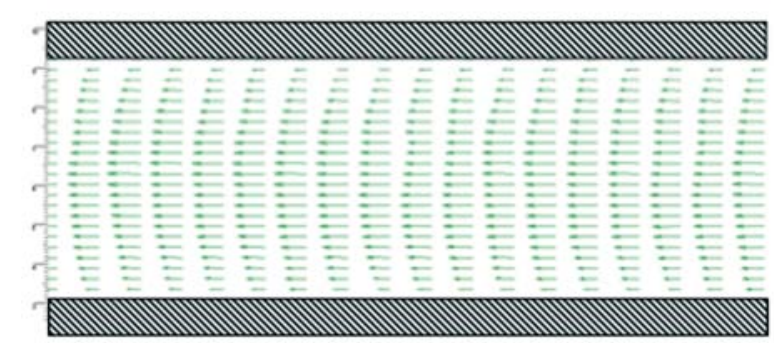

Figure 2. Typical experimental velocity field through the die (temperature: $280^{\circ} \mathrm{C}$, shear rate: $8.2 \mathrm{~s}^{-1}$ )

that the experiments were carried out on an industrial machine, whose temperature regulation is not perfect and may be slightly non-symmetric. Secondly, the speed is non-zero at the wall and can represent a significant part of the maximum speed for the lowest flow rates (Figure 3b). Thirdly, the profiles measured at shear rates above $13 \mathrm{~s}^{-1}$ have a bell shape instead of a pure parabolic one as it appears at lower flow rates.

The observations concerning wall slip are rather surprising if we consider the low shear rates $\left(<20 \mathrm{~s}^{-1}\right)$ involved in these experiments. Nevertheless, the measurements are relevant since the flow rate calculated from the velocity profile and the one obtained from melt weight measurements at the exit of the die show a good match (Figure 4). Wall slip has to be there to get this match. Furthermore, the measurements close to the wall fit perfectly to
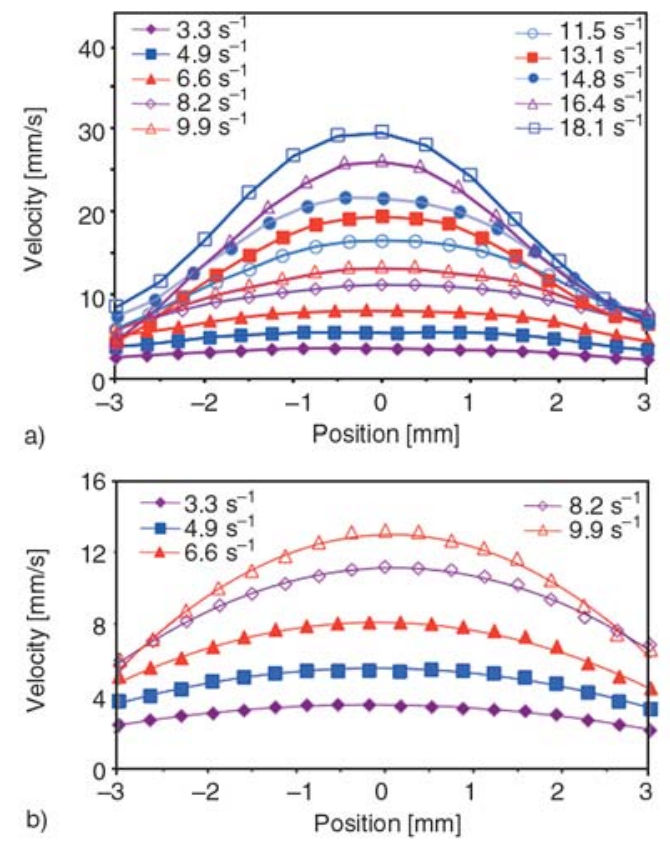

Figure 3. Flow profiles for various shear rates (temperature: $280^{\circ} \mathrm{C}$ ): a) whole series of profiles, b) detail of the profiles at low shear rates

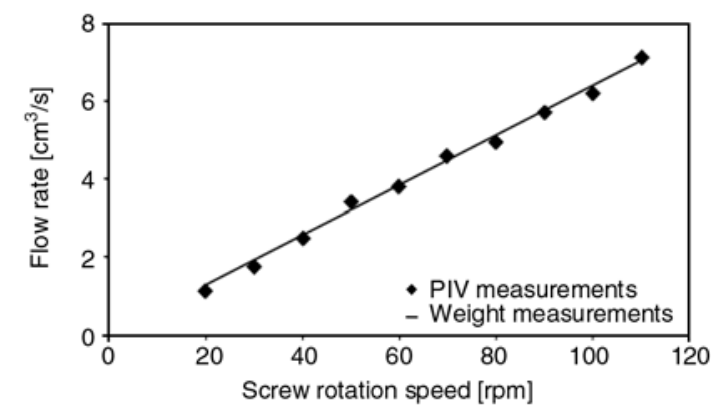

Figure 4. Comparison of the flow rates calculated from the velocity profiles and measured by melt weighing at the die outlet

the global velocity profile, and the same result would have been obtained by extrapolating the wall velocity from the central part of the profile. The measurements close to the wall are therefore reliable and we can fairly conclude that there is a significant slip at the wall within the spatial precision range of the measurement technique $(0.2-0.3 \mathrm{~mm})$. A non-slip layer thinner than the resolution of the system is obviously possible but such layer has not been reported in the literature even when 10 time more precise methods were used $[9,10]$.

Furthermore, similar tests were punctually carried out with PS. The analysis of the measured velocity fields (in the same flow rate range) does not show wall-slip phenomena, the measured velocity at wall being not significant $\left(<10^{-2} \mathrm{~mm} / \mathrm{s}\right)$. Rheometry measurements carried out with PS also confirm the sticky nature of the flow in that case. These results tend to confirm that the peculiarities of the velocity profiles observed with PC may effectively be ascribed to its ability to slip at the die wall.

The consequence of wall slip in case of polycarbonate is that the velocity profiles are sensibly different from what can be expected when making the nonslip assumption as it can be seen on Figure 5. The velocity gradient is lower when slip is present ('flatter' profiles), the maximum speed is reduced and the flow can be quite close to plug flow with slip speed as high as $70 \%$ of the maximum speed. This also influences the other parameters of the process, for example by a reduction in the die inlet pressure, as it will be reported in section 3.2.

The evolution of the wall slip vs. shear rate is given in Figure 6. It shows a linear evolution at low flow rates and then tends to stabilize. This evolution can be explained by two phenomena acting in opposite ways on the wall slip. Since the tests are performed 


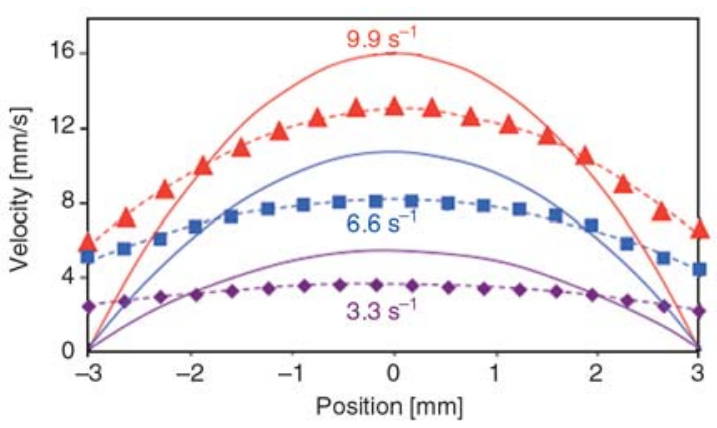

Figure 5. Experimental (symbols and dashed lines) vs. theoretical (solid line) velocity profiles through the die (Newtonian isothermal model between infinite parallel plates)

on an industrial extruder, the melt temperature is not fully controlled and depends on shear heating in the screw. Regarding the flowpath length, the viscous shear heating may become significant, contrary to what is observed on laboratory devices such as rheometers. As a consequence, the melt temperature rises when the screw rotation speed and therefore the flow rate are increased. Temperature measurements at the die exit showed a discrepancy of about $20^{\circ} \mathrm{C}$ between shear rates of 3 and $15 \mathrm{~s}^{-1}$ (Figure 6), whereas the shear heating induced temperature variations measured on the capillary rheometer remain lower than $2^{\circ} \mathrm{C}$. This shear heating, which is important in industrial configurations, should generate more slip [16-18]. On the other hand, the results are presented vs. shear rate and not shear stress. The rise in temperature comes together with a decrease in viscosity and shear stress, and therefore a lower slip. In the particular case of polycarbonate considered in this study, the first phenomenon appears to be dominant, and, for a given apparent shear rate at the die wall, the reduction of the apparent shear stress (and thus the wall slip velocity) linked to the viscosity

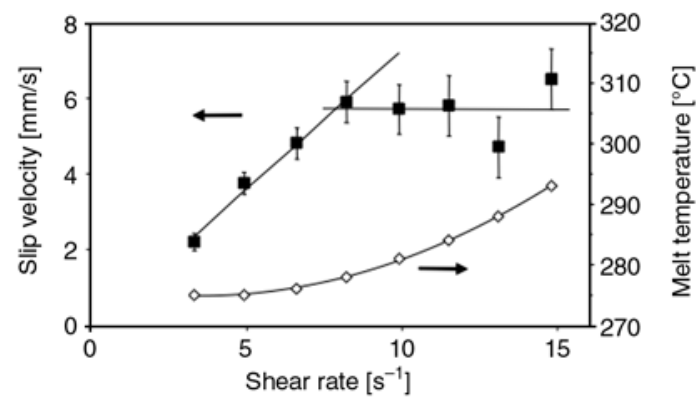

Figure 6. Wall slip velocity and melt temperature at the exit of the die vs. apparent shear rate (nominal temperature: $280^{\circ} \mathrm{C}$ ) decrease is not enough to compensate the increase of the slip velocity induced by the temperature elevation.

The distortion of the profiles at high flow rates (Figure 3a) can also be explained by thermal effects. Because of the shear heating of the melt (Figure 6), the temperature profile through the die is no longer isothermal when the flow rate is increased. This temperature gradient comes together with other gradients of physical properties of the melt, especially its density and its refractive index. The melt acts therefore as a lens and deforms the images that have to cross $30 \mathrm{~mm}$ of melt to exit the die and reach the recording camera. This was clearly visible at high flow rates because an apparent image of the die surfaces was visible away from the boundaries given by the steel surfaces. The higher the speed was, the narrower the apparent image was, which shows the temperature dependence of the phenomenon. This effect was partly corrected by resetting the walls position on the basis of their apparent image but it was impossible to have a perfect correction between these boundaries since the temperature gradient was unknown. This explains the peculiar shape of the velocity profiles. This is a physical phenomenon that cannot be avoided and that has to be taken into account when analyzing the results.

\subsubsection{Disrupted flows}

In a second phase, the PC flow was disrupted by introducing a geometrical discontinuity. The results obtained with this configuration are presented in Figures 7 and 8. Figure 7 presents the observation from the side of the die, similar point of view to the one used in the previous section. In Figure 8, laser lighting and recording camera were swapped in order to study the flow from above at two different levels. Note that the observation window and the obstacle are much narrower than the total width of the channel $(14 \mathrm{~mm}$ vs. $60 \mathrm{~mm})$ i.e. the polymer can flow beyond the observation window and the obstacle.

These measurements give a good description of the deviation of the flow when passing the discontinuity. One can see the effect of a large step but also of a small one. The lack of adjustment of the fixation screw generates a $0.5 \mathrm{~mm}$-deep depression on the surface of the obstacle. This creates waves and 


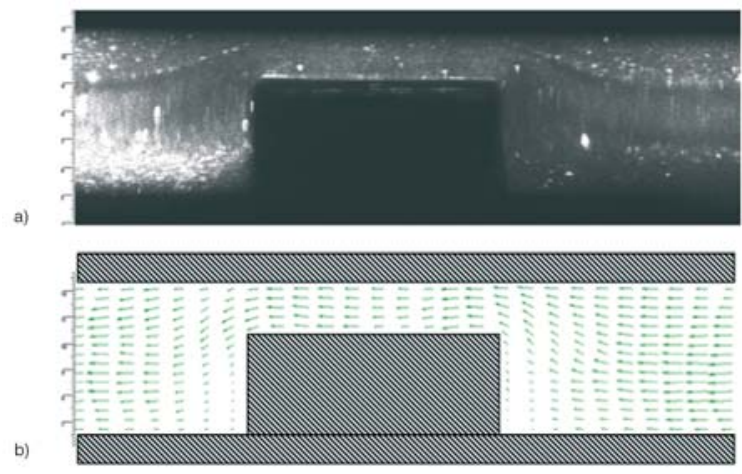

Figure 7. Velocity field in the vicinity of an obstacle (side view, flow from right to left): a) optical overview, b) velocity field

speed changes in the flow above the obstacle, which are recorded on the side view (Figure 7). The outline of this screw can also be distinguished on the top view (Figure 8c) through the distortion of the velocity field. The observation from two orthogonal points gives quantitative information about the three-dimensional flow. In the next section, these results will be compared to numerical simulations.

a)
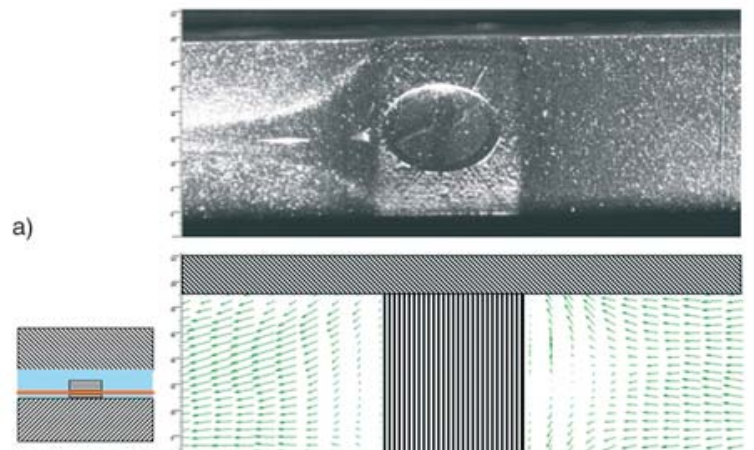

b)

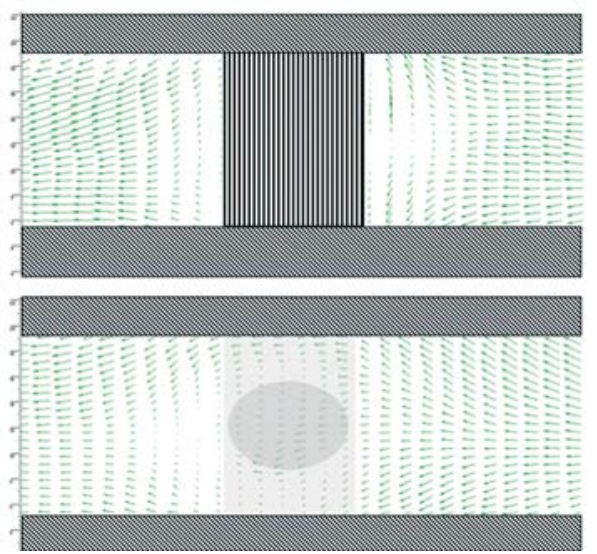

c)

Figure 8: Velocity field in the vicinity of an obstacle (top view, flow from right to left): a) optical overview, b) velocity field below the obstacle surface, $c$ ) velocity field above the obstacle

\subsection{Rheological measurements and numerical simulations}

Since wall slip was pointed out in section 3.1.1. for polycarbonate, this phenomenon was taken into account in the numerical simulations. The numeri-

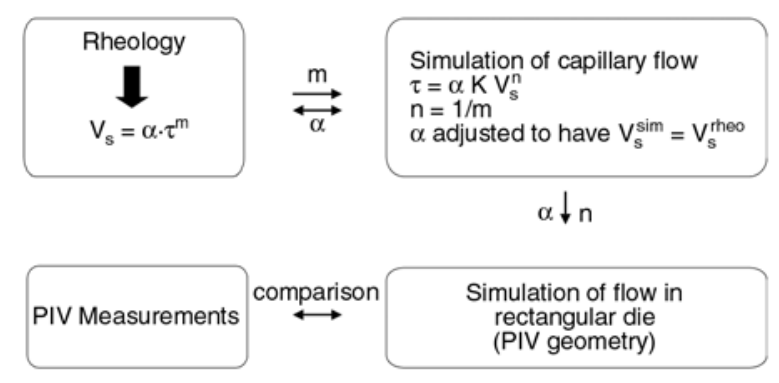

Figure 9. Combination of the different measurement and calculation results (for a given temperature)

cal slip parameters were determined using rheometry. Figure 9 gives an overview of the way the experimental results and numerical simulations were combined. The parameters of the numerical slip law were first determined on the basis of rheometry results, using the exponent $m$ as it was measured and adjusting the slip coefficient $\alpha$ in order to get a good match between the numerical and the rheological slip velocity when simulating a capillary flow. Numerical simulations were then performed on geometries corresponding to the PIV die using the slip parameters determined at the previous step.

Flow curves (Figure 10) were measured for different die diameters. Based on Mooney's method, the discrepancy between these curves was used to calculate the slip velocity using the slope of the curve obtained when plotting the apparent shear rate vs. the reverse diameter at constant shear stress. Figure 11 shows the evolution of the slip velocity with the apparent shear stress. One can see that slip is present and does not show any clear critical stress corresponding to the onset of slip down to $0.04 \mathrm{MPa}$, the flow curves showing a progressive departure from each other (Figure 10). Wall slip is connected to shear stress through a power law (Equation (4)) within the range of shear stress covered by this study $(0-0.18 \mathrm{MPa})$.

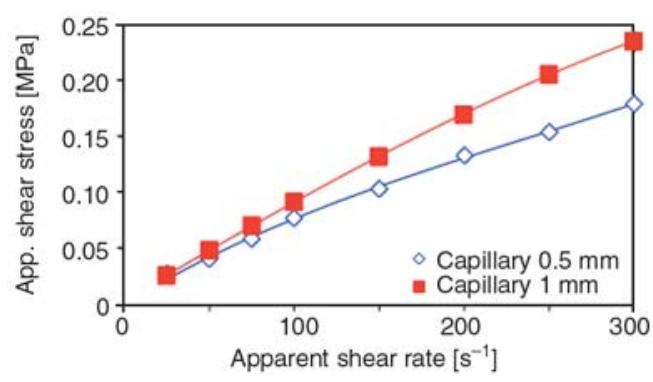

Figure 10. Flow curves for two capillaries diameters at $280^{\circ} \mathrm{C}$ 


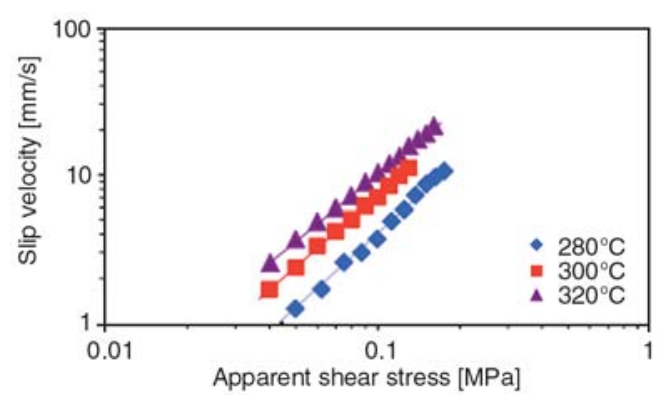

Figure 11. Slip velocity vs. apparent shear stress for different temperatures (capillary rheometry)

$V_{s}=\alpha \cdot \tau^{m}$

Table 1 reports the parameters of the law for different temperatures. It appears that temperature has a limited influence on the exponent $m$ whereas the coefficient $\alpha$ is strongly sensitive to it. For a given apparent shear stress, an increase in temperature induces a high increase in wall-slip velocity. For example, at an apparent shear stress of $0.1 \mathrm{MPa}$, the wall-slip velocity increases by $90 \%$ between 280 and $300^{\circ} \mathrm{C}$. The same trend was reported by Hatzikiriakos and Dealy $[16,17]$ in the case of high density polyethylene.

The ranges of shear rates used for capillary measurements (25-300 s $\left.\mathrm{s}^{-1}\right)$ and for PIV applied to extrusion $\left(<15 \mathrm{~s}^{-1}\right)$ are different, but it was unfortunately not possible neither to measure accurately enough the slipping velocity at lower shear rate on the capillary rheometer (limitation due to the pressure sensor resolution), nor to extrude at higher wall-shear rates (limitation due to the extruder capacity). However, the lower limit range of the shear rates used in capillary experiments $\left(25 \mathrm{~s}^{-1}\right)$ is not very far from the upper shear rates used in regular extrusion $\left(15 \mathrm{~s}^{-1}\right)$. These rheological experiments thus contribute to point out the existence of wall slipping, and to explain at least in term of general trend, the PIV measurements carried out on the industrial extrusion machine.

Figure 12 compares the experimental and simulated global velocity profiles through the thickness of the

Table 1. Parameters of the power law $\left(V_{s}=\alpha \cdot \tau^{\mathrm{m}}\right)$ describing the evolution of wall slip (in $\mathrm{mm} / \mathrm{s}$ ) vs. the apparent shear stress (in MPa) for different temperatures

\begin{tabular}{|c|c|c|}
\hline Temperature $\left[{ }^{\circ} \mathbf{C}\right]$ & $\boldsymbol{\alpha}\left[(\mathbf{m m} / \mathbf{s}) \cdot \mathbf{M P a}^{-\mathbf{m}}\right]$ & $\mathbf{m}[-]$ \\
\hline 280 & 186 & 1.65 \\
\hline 300 & 275 & 1.60 \\
\hline 320 & 316 & 1.50 \\
\hline
\end{tabular}

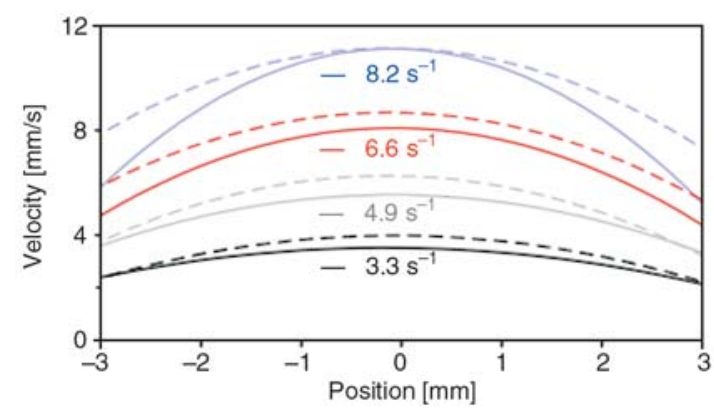

Figure 12. Experimental PIV velocity profiles through the thickness of the rectangular die (solid lines) and simulated profiles (dashed lines) for various shear rates

die. One can see that the fit is good for the lowest shear rates (3.3 and $\left.4.9 \mathrm{~s}^{-1}\right)$, whereas the discrepancy is more important, especially concerning the wall velocity, for the higher shear rates. This can be seen in Figure 13 that represents this velocity vs. apparent shear rate for simulation and experiment. One can see that the numerical results follow a power law as expected by the computing characteristics of the software. On the other hand, PIV measurements show a roughly linear evolution with shear rate and tend to stabilize above $8 \mathrm{~s}^{-1}$. This phenomenon was explained previously by thermal effects and the higher effect of temperaturedependence of wall slip compared to that of viscosity. The divergence between numerical simulation and direct observation by PIV can be attributed to the fact that the simulation software package takes into account the only temperature-dependence of viscosity, but disregards the temperature-dependence of the parameters of the slipping law. In order to confirm this hypothesis, some calculations have been performed as described hereafter. The polymer melt temperature used in the simulation has been increased (i.e. the value of the shear heating experimentally measured at the die exit (Figure 6) has been added to the polymer melt temperature). Then, the parameters of the slipping law have been

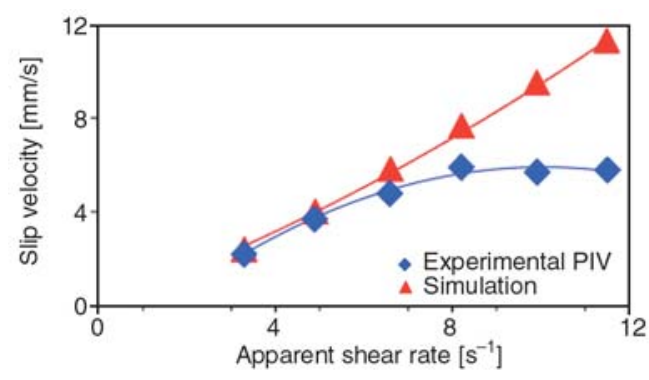

Figure 13. Experimental and numerical slip velocity vs. shear rate in the rectangular die at $280^{\circ} \mathrm{C}$ 


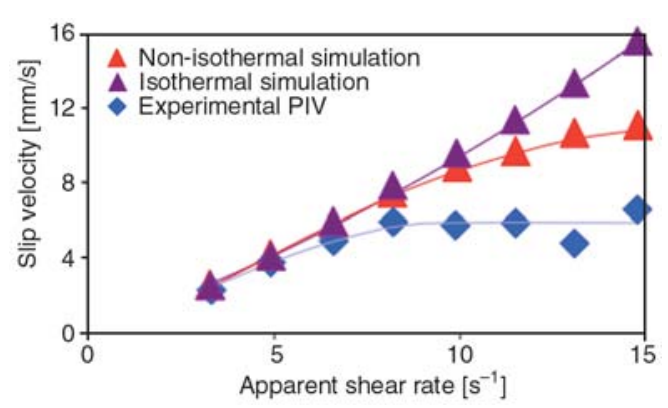

Figure 14. Slip velocity vs. apparent shear rate: influence of the increase in melt temperature on the simulated wall slip

adjusted accordingly by linear extrapolation between the rheological measurements at 280 and $300^{\circ} \mathrm{C}$. The simulated results obtained that way (Figure 14) are much closer to the experimental results, and also show the same wall-slip velocity stabilisation at higher shear rates as the one observed experimentally. Even if the difference between simulation and measurements still remains significant at higher shear rates, these results demonstrate that it is interesting and of prime importance to take the above-mentioned temperature-dependence in the slipping law.

Figure 15 represents the numerical velocity profiles with and without slip. It definitely appears that the velocity profile is significantly influenced by wall slip with reduction in the velocity range through the thickness and lower velocity gradients. Not taking slip into account can therefore lead to numerical simulations that are significantly different from the reality, not only in terms of velocity profiles but also concerning other important parameters of the process. For example, the inlet pressure is divided by two when wall slip is present (Figure 16).

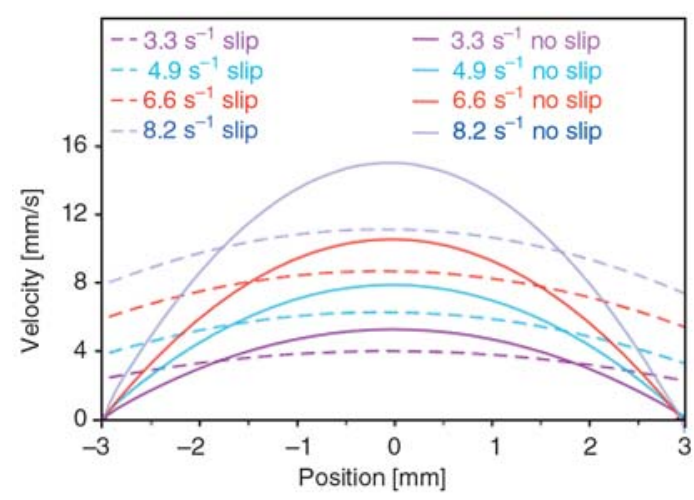

Figure 15. Numerical velocity profiles through the thickness of the die without (solid lines) and with (dashed lines) wall slip

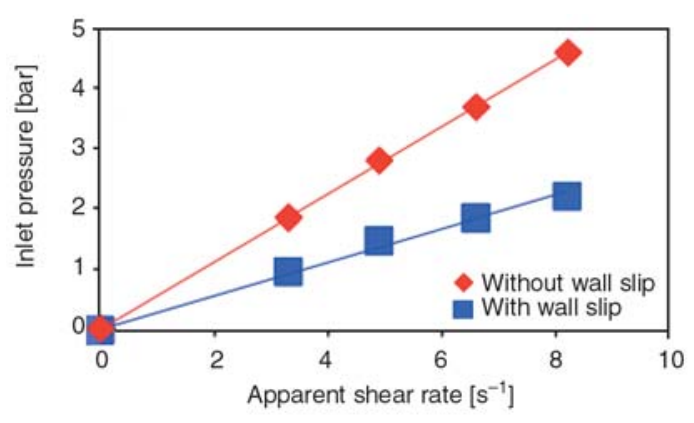

Figure 16. Simulated pressure at the entrance of the die vs. shear rate with and without wall slip

In a second step, the extrusion through the die with the geometrical discontinuity was simulated. Wall slip was introduced into the calculation using the same parameter as the one determined in the simple flow. Figure 17a shows the field of the x-component (main flow direction) of the velocity. The evolution of the speed globally as well as near the wall shows a good agreement with what can be observed in Figure 8. Wall speed changes in accordance with the local fluctuation of the stress due to passing of the complex obstacle.

Comparison with the non-slip case (Figure 17b) emphasizes the regulating effect of stick. The velocity fluctuations are much less pronounced in the absence of slip since the speed at the wall is always zero and is not sensitive to shear stress changes generated by the geometrical discontinuity. For example, the velocity in the center of the gap above the obstacle does not show more than $5 \%$ of variation along the obstacle in the stick condition whereas there is more than $30 \%$ decrease in velocity between the side and the center of the obstacle when wall slip is there. This shows that wall slip tends to destabilize the flow when passing geometrical singularities.

Figure 18 shows the flow lines around the discontinuity seen from the side and from above at two different levels. The agreement with Figures 7 and 8 is good, which proves the relevance of both experi-

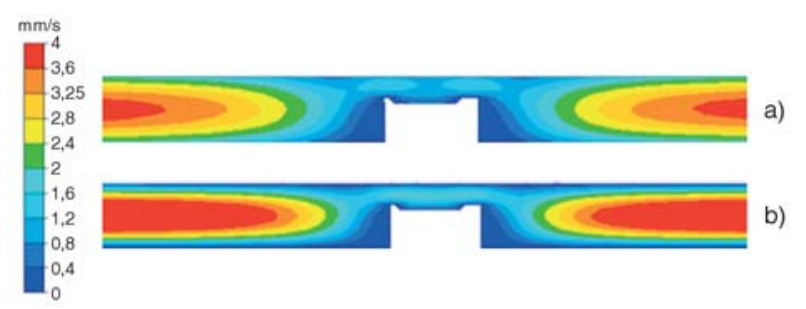

Figure 17. Simulated $x$-velocity field around an obstacle with (a) and without (b) wall slip (center plane, shear rate: $3.3 \mathrm{~s}^{-1}$ ) 


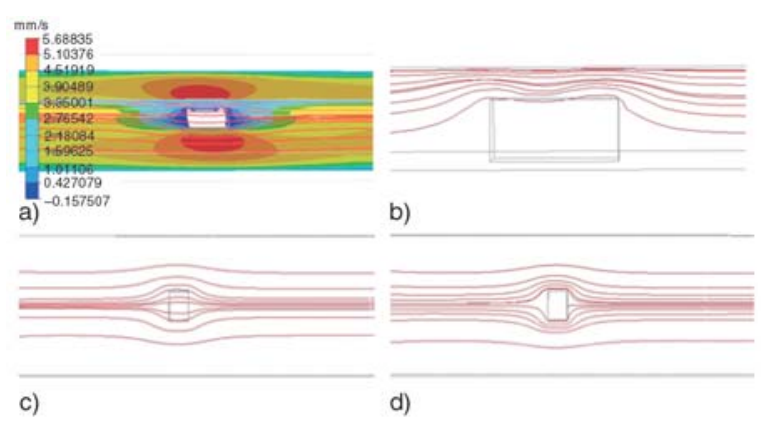

Figure 18. Simulated flow lines around an obstacle a) 3D overview (background: x-velocity map),

b) side view, c) top view above the obstacle,

d) top view below the obstacle surface

mental PIV measurements and numerical simulations. It is important to remember that the flow is complex with a real three dimensional behavior as shown in Figure 18a.

\section{Conclusions and outlook}

This study has shown the applicability of the PIV technique to characterize polymer flows in an extrusion die in very constraining conditions: real polymer (and not model fluid), industrial equipment (machine, tooling), high temperature, pressure and velocity conditions. PIV was successfully used to analyze the flow profiles globally as well as for a detailed analysis of the surface phenomena in the case of a particular reference of polycarbonate. Furthermore, it was possible to highlight an unusual behavior of the polycarbonate investigated, characterized by a significant wall-slipping even at very low shear rate, confirmed by rheometry measurements. Numerical simulations using slip parameters determined on the basis of simple rheometrical measurements showed a good agreement with experimental direct observation at low shear rate. At higher shear rate, the necessity to introduce a temperature-dependence of the slip parameters has been shown. This requires a more extensive characterization of the temperature influence and the development of the adequate models to account for its effect more accurately in the simulation.

Nonetheless, determining the numerical slip parameters on the basis of simple rheometrical measurements appeared, at least qualitatively, to be an interesting way to introduce wall slip in the simulation of flows in more complex geometries. PIV measurements validated this approach, showing a quite good agreement of the wall velocity in industrial equipment with numerical simulations.

\section{Acknowledgements}

This work has been partly funded by the French Ministère de l'Économie des Finances et de l'Industrie, as part of 'Réseau National Matériaux et Procédés', project 'Masther'. The authors also wish to thank Transvalor for supplying the simulation software.

\section{References}

[1] Yokoi H., Hayashi T., Morikita N., Todd K.: Direct observation of jetting phenomena under a high injection pressure by using a prismatic-glass inserted mould. in 'Proceedings of the SPE Annual Technical Conference ANTEC'88, Atlanta, USA' 329-333 (1988).

[2] Yokoi H., Murata Y., Oka K., Watanabe H.: Visual analysis of weld line vanishing process by glassinserted mould. in 'Proceedings of the SPE Annual Technical Conference ANTEC'91, Montreal, Canada' 367-371 (1991).

[3] Yokoi H., Nagami S., Kawasaki A., Murata Y.: Visual analysis of flow mark generation process using glassinserted mould. I. Micro-grooved flow marks. in 'Proceedings of the SPE Annual Technical Conference ANTEC'94, San Francisco, USA' 368-372 (1994).

[4] Yokoi H., Deguchi Y., Sakamoto I., Murata Y.: Visual analyses of flow mark generation process using glassinserted mould. II. Synchronous flow marks with same phases on both top and bottom surfaces of moulded samples. in 'Proceedings of the SPE Annual Technical Conference ANTEC'94, San Francisco, USA' 829-832 (1994).

[5] Yokoi H., Han X.: Visualization analysis of melt flowing behavior into micro-scale grooves during cavity filling process in injection molding. in 'Proceedings of the $21^{\text {st }}$ Annual Meeting of the Polymer Processing Society, Leipzig, Germany' CD-ROM, SL 2.6, p8 (2005).

[6] Dowling D. R., Bress T. J.: Visualisation of injection moulding. in 'Proceedings of the SPE Annual Technical Conference ANTEC'97, Toronto, Canada' Vol III, 3692-3696 (1997).

[7] Nabialek J.: Monitoring and recording of the polymer flow in a mold cavity during the injection molding process. in 'Proceedings of the $21^{\text {st }}$ Annual Meeting of the Polymer Processing Society (PPS-21), Leipzig, Germany' Paper P 13.7 (2005). 
[8] Agassant J-F., Arda D., Combeaud C., Merten A., Münstedt H., Mackley M. R., Robert L., Vergne B.: Polymer processing extrusion instabilities and methods for their elimination or minimization. in 'Proceedings of the $21^{\text {st }}$ Annual Meeting of the Polymer Processing Society (PPS-21), Leipzig, Germany' Paper KL 1.3 (2005).

[9] Münstedt H., Schmidt M., Wassner E.: Stick and slip phenomena during extrusion of polyethylene melts as investigated by laser-Doppler velocimetry. Journal of Rheology, 44, 413-427 (2000). DOI: $10.1122 / 1.551092$

[10] Robert L., Demay Y., Vergnes B.: Stick-slip flow of high density polyethylene in a transparent slit die by laser Doppler velocimetry. Rheologica Acta, 43, 89-98 (2004).

DOI: $10.1007 / \mathrm{s} 00397-003-0323-\mathrm{x}$

[11] Mitsoulis E., Schwetz M., Münstedt H.: Entry flow of LDPE melts in a planar contraction. Journal of NonNewtonian Fluid Mechanics, 111, 41-61 (2003). DOI: $10.1016 / \mathrm{S} 0377-0257(03) 00012-0$

[12] Martyn M. T., Gough T., Spares S., Coates P. D., Zarloukal M.: Visualisation and analysis of LDPE melt flows in a coextrusion geometry. in 'Proceedings of the SPE Annual Technical Conference ANTEC 2002, San Francisco, USA' 937-941 (2002).
[13] Yokoi H., Inagaki Y.: Dynamic visualisation of cavity filling process along the thickness direction using a laser light sheet technique. in 'Proceedings of the SPE Annual technical Conference ANTEC'92, Detroit, USA' 457-460 (1992).

[14] Nigen S., El Kissi N., Piau J. M., Sadun S.: Velocity field for polymer melts extrusion using particle image velocimetry: Stable and unstable flow regimes. Journal of Non-Newtonian Fluid Mechanics, 112, 177202 (2003).

DOI: $10.1016 / \mathrm{S} 0377-0257(03) 00097-1$

[15] Buchhave P.: Particle image velocimetry - status and trends. Experimentals Thermal and Fluid Science, 5, 586-604 (1992). DOI: $\underline{10.1016 / 0894-1777(92) 90016-X}$

[16] Hatzikiriakos S. G., Dealy J. M.: Wall slip of molten high density polyethylene I. Sliding plate rheometer studies. Journal of Rheology, 35, 497-523 (1991). DOI: $10.1122 / 1.550178$

[17] Hatzikiriakos S. G., Dealy J. M.: Wall slip of molten HDPE. II. Capillary rheometer studies. Journal of Rheology, 36, 703-741 (1992). DOI: $10.1122 / 1.550313$

[18] Maciel A., Salas V., Soltero J. F. A., Guzman J., Manero O.: On the wall slip of polymer blends. Journal of Polymer Science Part B: Polymer Physics, 40, 303-316 (2002). DOI: $10.1002 /$ polb.10093 